\title{
Atividades de investigação em Cálculo Diferencial e Integral: uma proposta para o ensino do conceito de limite de uma função com o software GeoGebra
}

\author{
Research Activities in Differential and Integral Calculus: a proposal for teaching the \\ concept of a function limit with the GeoGebra software
}

\author{
Tailon Thiele \\ Universidade do Porto (UP), Faculdade de Ciências, Departamento de Matemática \\ Porto, Portugal \\ Universidade Regional Integrada do Alto Uruguai e das Missões (URI) \\ Frederico Westphalen, RS, Brasil \\ https://orcid.org/0000-0003-4453-9852, thiele.tailon@gmail.com
}

Eliane Miotto Kamphorst Universidade Regional Integrada do Alto Uruguai e das Missões (URI) Frederico Westphalen, RS, Brasil

https://orcid.org/0000-0002-0302-5700, miottokamphorst@gmail.com

Carmo Henrique Kamphorst Instituto Federal de Educação, Ciência e Tecnologia Farroupilha (IFFar)

Frederico Westphalen, RS, Brasil

https://orcid.org/0000-0002-5609-7943, carmohenriquek@gmail.com

\section{Informações do Artigo \\ Como citar este artigo \\ THIELE, Tailon; KAMPHORST, Eliane Miotto; KAMPHORST, Carmo Henrique. Atividades de investigação em Cálculo Diferencial e Integral: uma proposta para o ensino do conceito de limite de uma função com o software GeoGebra. REMAT: Revista Eletrônica da Matemática, Bento Gonçalves, RS, v. 6, n. 2, p. e2002, 15 ago. 2020. DOI: \\ https://doi.org/10.35819/remat2020v6i2id3987}

Histórico do Artigo

Submissão: 27 de março de 2020

Aceite: 25 de abril de 2020.

\section{Resumo}

Este trabalho objetiva apresentar algumas considerações acerca do desenvolvimento de atividades investigativas apoiadas na utilização de Tecnologias Digitais de Informação e Comunicação (TDIC), no ensino de Cálculo Diferencial e Integral, além de uma proposta metodológica para o ensino do conceito de limite de uma função. Para isso, realizaram-se diversos apontamentos e considerações a partir do existente na bibliografia, sobre a importância da elaboração de atividades de investigação matemática a partir do uso de tecnologias. Salienta- se, ainda, a reconfiguração do papel docente, que precisa estar preparado para lidar em uma organização dinâmica do espaço de aprendizagem. Por fim, é feito um estudo exploratório no qual é apresentada e discutida uma proposta de ensino, baseada na investigação matemática em sala de aula, para a abordagem do conceito de limite de uma função com o uso do software GeoGebra.

\section{Palavras-chave}

Investigação Matemática

Tecnologias Digitais

Aprendizagem Conceitual

Cálculo Diferencial e Integral

\section{Keywords}

Mathematical Research

Digital Technologies

Conceptual Learning

Differential and Integral Calculus

\begin{abstract}
This work aims to present some considerations about the development of investigative activities supported on the use of Digital Technologies of Information and Communication (TDIC), in the teaching of Differential and Integral Calculus, besides a methodological proposal for the teaching of the concept of limit of a function. For this, several notes and considerations were made based on what exists in the bibliography, on the importance of
\end{abstract}


developing mathematical research activities based on the use of technologies. It is also worth highlighting the reconfiguration of the teaching role, which needs to be prepared to deal with a dynamic organization of the learning space. Finally, an exploratory study is carried out in which a teaching proposal is presented and discussed, based on mathematical research in the classroom, to approach the concept of limit of a function using the GeoGebra software.

\section{Introdução}

Novas perspectivas têm norteado a educação, especialmente a Educação Matemática, a fim de buscar formas de ensino e aprendizagem voltadas à construção do conhecimento. Dentre essas perspectivas, o desenvolvimento de atividades investigativas no processo de ensino e aprendizagem de conceitos matemáticos vem construindo diferentes concepções acerca dos currículos e do papel do professor, tanto na Educação Básica, quando no Ensino Superior (BORBA; PENTEADO, 2012). A estrutura do currículo deve buscar a formação de capacidades intelectuais, estruturação do pensamento e o raciocínio lógico (MOTTA et al., 2011).

Entretanto, no âmbito de cursos superiores das áreas de Ciências Exatas e da Terra e Engenharias, em decorrência de abordagens metodológicas tradicionais, geralmente caracterizadas pela transmissão de conceitos e resolução de exercícios fechados com ênfase no treinamento algébrico, são apontados índices alarmantes de reprovação e evasão na disciplina de Cálculo Diferencial e Integral (COSTA; OLIVEIRA, 2020, VIEIRA; RIOS; VASCONCELOS, 2019, WISLAND; FREITAS; ISHIDA, 2014, PAGANI; ALLEVATO, 2014, GARZELLA, 2013, RESENDE, 2003, BARUFI, 1999). Nesse sentido, reforça-se a concepção de que é preciso construir significados conceituais por meio de aplicações norteadas pela investigação, de modo que os estudantes estejam cientes da importância dessa disciplina e envolvidos com a aprendizagem (RESENDE, 2003).

Tendo em vista essa compreensão, justifica-se a construção de aportes teóricometodológicos sobre as novas perspectivas para o ambiente de aprendizagem. $\mathrm{O}$ emprego de atividades de investigação matemática aliadas à incorporação de ferramentas tecnológicas estão entre essas possibilidades, dadas as suas contribuições para uma aprendizagem mais significativa. É importante salientar que aprendizagem significativa, segundo Ausubel (2003), o processo de construção de esquemas de pensamento que relacionam conceitos novos e anteriores, sem que haja um isolamento de conteúdos. A partir disso, os papeis de professor e alunos se reconstroem, especialmente no sentido da participação efetiva do estudante na sua aprendizagem.

No entanto, a complexidade desse processo vincula-se à necessidade de uma mudança sistemática nos paradigmas educacionais propostos atualmente. É preciso repensar a organização das instituições, de modo que os agentes que participam do seu funcionamento 
tenham claros os objetivos da educação democrática, e de suas atribuições nesse contexto. Da mesma forma, esta transformação perpassa pelos recursos materiais, até a atuação docente e o estudante. Qualificar a educação requer objetivos em comuns e relações de respeito com o eu, com o outro e com meio ambiente (LUCKESI, 2007).

Nesta perspectiva, a construção e incorporação de abordagens metodológicas diferenciadas pressupõem o conhecimento acerca do papel da disciplina na formação profissional do estudante, de acordo com as relações que lhe são atribuídas. A partir desse processo, surgem proposições didáticas e ferramentas inovadoras, que podem nortear o trabalho docente na organização pedagógica. Dentre essas propostas, as investigações matemáticas aliadas à incorporação de TDIC são importantes possibilidades na busca por aprendizagens mais significativas (BARBOSA, 2001).

Dados esses apontamentos, o presente artigo tem por objetivo apresentar algumas considerações acerca do desenvolvimento de atividades investigativas apoiadas na utilização de TDIC, para uma aprendizagem significativa na disciplina de Cálculo Diferencial e Integral. Além disso, é apresentada uma proposta metodológica para o ensino do conceito de limite de uma função no software GeoGebra, por meio de uma atividade investigativa, a fim de enriquecer as discussões sobre as formas de organização didática, e que poderá ser incorporada por docentes nos espaços em que atuam.

\section{Referencial teórico}

\subsection{Aprendizagem significativa}

Os processos de ensino e aprendizagem estão entrelaçados de tal modo que um influencia o outro e vice-versa. Isto é, o ensino e as suas formas de abordagem interferem diretamente na aprendizagem dos estudantes, de forma que é necessário intervir no processo pedagógico a partir da identificação de possíveis dificuldades na aquisição de conhecimentos. Portanto, o trabalho docente deve estar estruturado com base no que se observa na aprendizagem.

A aprendizagem significativa se trata de um conceito trabalhado por Ausubel (2003), a partir de um enfoque cognitivo.

[...] A aquisição de novos significados a partir de material de aprendizagem apresentado. Exige quer um mecanismo de aprendizagem significativa, quer a apresentação de material potencialmente significativo para o aprendiz. Por sua vez, a última condição pressupõe (1) que o próprio material de aprendizagem possa estar relacionado de forma não arbitrária (plausível, sensível e não aleatória) e não literal com qualquer estrutura cognitiva apropriada e relevante (i.e., que possui significado 'lógico') e (2) que a estrutura cognitiva particular do aprendiz contenha ideias ancoradas relevantes, com as quais se possa relacionar o novo material. A interação entre novos significados potenciais e ideias relevantes na estrutura cognitiva do aprendiz dá origem a significados verdadeiros ou psicológicos. Devido à estrutura cognitiva de cada aprendiz ser única, todos os novos significados adquiridos são, também eles, obrigatoriamente únicos (AUSUBEL, 2003, p. 17). 
Portanto, aprender significativamente requer uma base de conhecimentos em que os novos conceitos serão incorporados. Trata-se da elaboração e organização de esquemas de pensamento que relacionem conhecimentos prévios e novos, sem que aconteça um isolamento de conteúdos. Nesse processo, são atribuídos significados aos novos conteúdos, bem como são construídas novas concepções acerca dos conceitos já existentes na memória do aprendiz. De forma simplificada, é a interação entre conhecimentos novos e antigos (AUSUBEL, 2003).

Outra ideia importante tratada pela teoria de Ausubel (2003) é a aprendizagem conceitual. Segundo o autor, existem dois métodos gerais de aprendizagem conceitual. O primeiro refere-se à formação conceitual usada por crianças no início de sua formação de ideias. Já o segundo, é o método utilizado por crianças em idade escolar e indivíduos adultos, caracterizado pela assimilação conceitual. Isto é,

\begin{abstract}
$\mathrm{Na}$ formação conceitual, os atributos específicos do conceito adquirem-se através de experiências diretas, através de fases sucessivas de formulação de hipóteses, testes e generalização. Contudo, à medida que o vocabulário de uma criança aumenta, adquirem-se novos conceitos, sobretudo através do processo de assimilação conceitual, visto que os atributos específicos dos novos conceitos se podem definir com a utilização em novas combinações de referentes existentes, disponíveis na estrutura cognitiva da criança (AUSUBEL, 2003, p. 18).
\end{abstract}

Nesse sentido, pretende-se pensar na aprendizagem significativa e aprendizagem de conceitos na Educação Matemática a partir da elaboração de situações em que o aprendiz se coloque diante da possibilidade de investigar novos conceitos com base no seu aporte de conhecimentos já existente. Entende-se, então, que a investigação de conceitos matemáticos pode estar embasada na utilização de tecnologias digitais e isto é discutido a seguir.

\title{
2.2. Considerações acerca do desenvolvimento de investigação matemática $e$ incorporação de tecnologias digitais no ambiente de aprendizagem de Cálculo Diferencial e Integral
}

Os processos de ensino e aprendizagem têm recebido ênfase por pesquisadores da área de Educação Matemática, especialmente na busca por alternativas pedagógicas que coloquem o estudante em evidência. No espaço universitário, é importante que a forma de abordagem metodológica permita relacionar os conceitos com situações práticas, de modo que os discentes reconheçam os problemas como pertencentes de suas áreas de atuação, no contexto pragmático (SKOVSMOSE, 2001).

O estudante do curso universitário está em busca de uma formação sólida que o capacite para o mercado de trabalho. Dessa forma, os alunos procuram um conhecimento que seja útil em sua futura vida profissional. Considerando esse perfil, é importante que o estudante conheça onde se aplicam os conceitos trabalhados pelo professor de Cálculo, para que os conteúdos estudados passem a ter algum significado prático (GONÇALVES; REIS, 2013, p. 421).

Dada esta importância de uma aprendizagem significativa e contextualizada, os docentes necessitam buscar ferramentas e métodos que tornem o estudante partícipe no ambiente de 
aprendizagem. É imprescindível que o aluno construa pensamento próprio, elabore relações entre os objetos matemáticos e suas propriedades, além de observar regularidades na resolução de problemas por meio de atividades investigativas (GONÇALVES; REIS, 2013). Ainda, a contextualização de saberes

[...] é uma ferramenta indispensável para a questão da transposição didática, pois implica recorrer a contextos que tenham significado para o aluno, envolvendo-o não só intelectualmente, mas também afetivamente, sendo assim uma estratégia fundamental para a construção de significados. Sabemos que a falta de sentido da aprendizagem de Cálculo Diferencial e Integral origina-se, em parte, das dificuldades decorrentes dessa transposição. $O$ aluno só compreende os vínculos do conteúdo estudado quando fica compreensível para ele essa passagem. Por isso, contextualizar no ensino de Cálculo vincularia os conhecimentos aos lugares onde foram criados e onde são aplicados, isto é, incorporar vivências concretas ao que se vai aprender e incorporando o aprendizado a novas vivências (BARBOSA, 2004, p. 41).

Conforme a ideia apresentada por Ausubel (2003) acerca da aprendizagem conceitual, entende-se que as ferramentas e abordagens metodológicas utilizadas pelos docentes do Ensino Superior, especialmente na disciplina de Cálculo, influenciam diretamentepara que ela aconteça. A utilização de atividades que possibilitam a investigação permite a discussão e contextualização com problemas da área de futura atuação, de modo que o conhecimento seja útil e interessante ao aluno, aumentando o seu valor em relação à importância. Além disso, atividades de investigação tornam o aluno ativo no ambiente de aprendizagem, despertando sua criatividade e, principalmente, sua autonomia sobre a aprendizagem (SILVA; VERTUAN, 2018).

Salienta-se, desse modo, que a investigação matemática acontece a partir de problemas abertos, em que a situação não está bem definida. Cabe ao investigador o papel de interpretar e buscar soluções. Sendo assim, é possível haver diferentes compreensões, sem que, necessariamente, exista apenas uma correta. A resolução, por sua vez, não perpassa apenas pela aplicação de métodos já conhecidos. A participação ativa do estudante na formulação de ideias acerca dos problemas matemáticos é fundamental para que ele mobilize seus recursos cognitivos e afetivos e, esse processo, é imprescindível para a aprendizagem (PONTE; BROCARDO; OLIVEIRA, 2003).

O conceito de investigação matemática, como atividade de ensino-aprendizagem, ajuda a trazer para a sala de aula o espírito da atividade matemática genuína, constituindo, por isso, uma poderosa metáfora educativa. O aluno é chamado a agir como um matemático, não só na formulação de questões e conjecturas e na realização de provas e refutações, mas também na apresentação de resultados e na discussão e argumentação com os seus colegas e o professor (PONTE; BROCARDO; OLIVEIRA, 2003, p. 23).

O papel docente nesta perspectiva toma três dimensões distintas: organizar, facilitar e mediar. A primeira dimensão requer a organização de situações de aprendizagem que proporcionem a construção de conceitos e procedimentos pelo educando. Na segunda dimensão, o professor é facilitador da aprendizagem, pois é o responsável pela construção do conhecimento que o aluno não consegue obter sozinho. Já o professor enquanto mediador da aprendizagem orienta e intervém na aprendizagem quando for necessário (MOTTA et al., 2011). O professor 
deve elaborar atividades que despertem o interesse do aluno, para que este se envolva na aplicação de conceitos matemáticos. Isso requer uma participação efetiva, porém, como intermediário entre aluno e produção de conhecimento (SANTOS; BELLINE, 2016).

$O$ professor lança questões desafiadoras e ajuda os alunos a se apoiarem, uns nos outros, para atravessarem as dificuldades. O professor faz a intermediação, leva os alunos a pensar, espera que eles pensem, dá tempo para isso, acompanha suas explorações e resolve, quando necessário, problemas secundários (ONUCHIC, 1999, p. 216).

No entanto, investigar conceitos matemáticos pressupõe a utilização de ferramentas capazes de enriquecer as possibilidades de exploração do conhecimento. Destaca-se, então, a utilização das TDIC, tendo em vista os apontamentos favoráveis em relação à sua incorporação no ambiente de aprendizagem. Trata-se de democratizar os processos educativos, de modo que os estudantes tenham acesso a novas linguagens e formas de raciocínio, com base na experimentação e simulação de processos e conceitos matemáticos (BORBA; PENTEADO, 2012). Isto está estreitamente relacionado com o conceito de aprendizagem significativa de Ausubel (2003), pois,

As tecnologias propiciam investigações matemáticas, pois, com uma única atividade podem emergir outras perguntas, problemas, observação de regularidades, investigações e outros conceitos podem ser retomados ou abordados. Além disso, o professor de Cálculo tem aí uma possibilidade de tornar a abordagem de certos conceitos significativa para os estudantes, gerando novas compreensões em função da ampliação das formas de interação aluno-conteúdo, comparando-se com estratégias metodológicas clássicas, que priorizam a abordagem estática do conteúdo (RICHIT; FARIAS, 2013, p. 5).

Dessa forma, é relevante que a prática pedagógica esteja baseada em atividades de investigação matemática, e que esse processo se encontra vinculado à utilização de ferramentas tecnológicas digitais. Daí a necessidade de estudos que abordem conceitos específicos de Cálculo Diferencial e Integral, na busca por amenizar a problemática apresentada na introdução desde trabalho.

\subsection{Proposição de uma atividade para o ensino de Cálculo Diferencial e Integral: apresentação e justificativas}

Apesar de todas as considerações descritas anteriormente, o trabalho pedagógico na disciplina de Cálculo Diferencial e Integral ainda carece de atividades de investigação de conceitos, especialmente com a utilização de tecnologias digitais, conforme vem sendo abordado por diversos autores nos últimos anos (COSTA; OLIVEIRA, 2020, VIEIRA; RIOS; VASCONCELOS, 2019, WISLAND; FREITAS; ISHIDA, 2014, PAGANI; ALLEVATO, 2014, GARZELLA, 2013, RESENDE, 2003, BARUFI, 1999). Como consequência, os mesmos autores citam dificuldades de aprendizagem e altos índices de reprovação e evasão.

Dada essa problemática, tem-se aqui a intenção de pensar o trabalho pedagógico em Cálculo Diferencial e Integral a partir das contribuições da investigação matemática que foram 
apresentadas. É preciso entender a formação de nível superior como a construção de sujeitos com identidades próprias e capazes de estabelecer conexões entre o conhecimento teórico e o contexto pragmático de futura atuação profissional. Entendemos que, para que isso aconteça, é imprescindível que o ambiente de aprendizagem forneça condições para que os estudantes compreendam conceitos, e não apenas apliquem propriedades operatórias de forma mecanizada.

A partir dessa concepção, justifica-se a elaboração de propostas pedagógicas que tomem como porto de partida os conceitos de aprendizagem significativa e conceitual (AUSUBEL, 2003), e levem em consideração as contribuições da incorporação de investigação matemática aliada ao uso de tecnologias digitais para o ensino de conceitos de Cálculo Diferencial e Integral. A intenção deste trabalho não é descrever propriedades operatórias para a resolução de problemas matemáticos. Contrariamente, pretende-se apresentar uma proposta com potencial para que os estudantes consigam desenvolver uma noção intuitiva acerca de um conceito.

Optou-se neste artigo por abordar o conceito de limite de uma função, por ser, geralmente, o primeiro conceito fundamental estudado na disciplina de Cálculo Diferencial e Integral. Uma parcela significativa de estudantes chega ao Ensino Superior com ideias fragmentadas da Matemática básica, e pode estar diante de várias dificuldades no estudo dos conceitos do Ensino Superior. Por isso, é importante que as formas de abordagem possibilitem condições de aprendizagem mais dinâmicas. Além disso, conforme é destacado por Amorim, Sousa e Salazar (2011), esses conceitos possuem sua epistemologia geométrica, mas que acabou sendo deixada de lado pelos docentes. Nesse sentido, buscar-se-á aqui pensar o conceito de limite a partir de uma forma dinâmica.

\subsection{O limite de uma função}

O conceito de limite de uma função, de modo geral, é o primeiro a ser trabalhado no estudo de Cálculo Diferencial e Integral, pois está associado à compreensão de outros conceitos que o sucedem. Stewart (2012), define o limite de uma função escrevendo $\lim _{x \rightarrow a} f(x)=L$, onde lêse

"o limite de $f(x)$, quando $x$ tende a $a$, é igual a $L$ ", se pudermos tornar os valores de $f(x)$ arbitrariamente próximos de $L$ (tão próximos de $L$ quanto quisermos), tomando $x$ suficientemente próximo de $a$ (por ambos os lados de $a$ ), mas não igual a $a$ (STEWART, 2012, p. 78).

Existem diferentes formas de abordagem e que utilizam distintas representações para o conceito de limite. Fuente, Armenteros e Moll (2012) apresentam cinco significações: gráfica, geométrica, pré-infinitesimal, infinitesimal e numérica. Obviamente, todas essas compreensões possuem conexão com o conceito geral apresentado no parágrafo anterior. O significado gráfico está relacionado à representação gráfica de funções e o conceito de limite. O significado geométrico refere-se ao axioma da continuidade. O pré-infinitesimal requer um raciocínio voltado ao "infinitamente pequeno" e o limite no infinito. A ideia infinitesimal corresponde a substituir o 
valor ao qual o limite tente, e o pensamento abstrato de aproximação. Já o significado numérico está voltado à elaboração de tabelas com valores à variável independente.

$\mathrm{Na}$ abordagem proposta neste trabalho, estão abordados três desses significados (nomeadamente: gráfico, geométrico e infinitesimal). No entanto, essas representações não são tratadas de forma dissociada. Reconhece-se a importância dos demais significados, sugerindo-se que o leitor possa adaptar a sequência didática de acordo com os objetivos da aula, bem como acrescentar se lhe for conveniente.

\subsection{O software GeoGebra}

O GeoGebra apresenta várias possibilidades de estudo a partir de uma interface que faz uma conexão entre a Álgebra e a Geometria, de modo que sejam realizadas investigações e a compreensão de diferentes registros de representação do objeto matemático. Borba, Silva e Gadanidis (2014) discutem sobre a evolução das tecnologias digitais na Educação Matemática, dividindo-a em quatro fases, que partem do uso de computadores e calculadoras simples, até a dinamicidade digital e interatividade, no momento atual. Os autores situam o GeoGebra dentro dessas últimas características, evidenciando o seu valor pedagógico dentro das ferramentas tecnológicas disponíveis.

Além disso, Jardim et al. (2015) destacam que é necessário possibilitar a condição de participação ativa ao estudante, uma vez que apenas visualizações abstratas não são suficientes para uma boa compreensão do conteúdo. Isso fica mais evidente no estudo de limites, pois muitas vezes é o primeiro contato dos estudantes com a Matemática do Ensino Superior. Segundo os autores, o GeoGebra é uma boa alternativa para atividades exploratórias.

Outra vantagem que deve ser destacada, e que não diz respeito ao seu valor pedagógico, é que se trata de uma ferramenta de acesso livre, de modo a permitir que qualquer estudante ou professor possa facilmente tê-lo em seu próprio dispositivo eletrônico. As versões originais podem ser baixadas em https://www.geogebra.org/download. Neste trabalho, a versão utilizada pelos autores é a "GeoGebra Clássico 5".

\subsection{Algumas propostas para abordagem do conceito de limite com o GeoGebra disponíveis na literatura}

O ensino de limites com o auxílio do software GeoGebra vem sendo abordado em alguns trabalhos já publicados. Jardim et al. (2015) propõem um modelo de atividade na forma de "quiz", em que os estudantes são desafiados a analisar o gráfico da função e responder algumas questões específicas acerca do seu comportamento em relação ao limite. Além disso, os autores acrescentam a possibilidade do estudo do limite a partir de controles deslizantes.

Silva e Becker (2018) apresentam uma proposta metodológica do estudo do limite com o GeoGebra a partir de um objeto de aprendizagem. Nesse modelo, a investigação é feita de modo 
mais abstrato, a partir da aplicação do conceito em problemas da geometria, especificamente comprimento e área. Fonseca e Henriques (2016), também sugerem a utilização do GeoGebra no ensino de limites. Esses autores relatam resultados positivos de uma atividade realizada em um curso de formação inicial de professores de Matemática.

Como é notável, as propostas citadas anteriormente foram publicadas recentemente, evidenciando que ainda há caminhos para se percorrer quanto a um melhor aproveitamento desta ferramenta no ambiente de aprendizagem, especificamente no estudo de limites. Isso reforça a importância do desenvolvimento de mais trabalhos com propostas metodológicas com potencial pedagógico, atingindo cada vez mais docentes de Cálculo Diferencial e Integral e demais interessados.

A proposta apresentada a seguir também apresenta uma forma de abordagem exploratória do conceito de limite de uma função no GeoGebra. Em comparação com as ideias de Jardim et al. (2015), Silva e Becker (2018), e Fonseca e Henriques (2016), pretende-se dar ainda mais autonomia aos estudantes, especialmente a partir de uma atividade construída inteiramente pelos próprios alunos. A intenção não está restrita a responder questões específicas, mas sim em fornecer a possibilidade de um entendimento amplo e intuitivo do conceito de limite, qualquer que seja a função analisada.

\section{Proposta metodológica para o ensino de limite}

Na Figura 1 é apresentada uma imagem geral da interface inicial do software. Inicialmente, é possível visualizar algumas ferramentas básicas como a janela de álgebra, a janela de visualização gráfica, barra de menus, barra de ferramentas e a janela de entrada de comandos.

Figura 1 - Interface inicial do software GeoGebra.

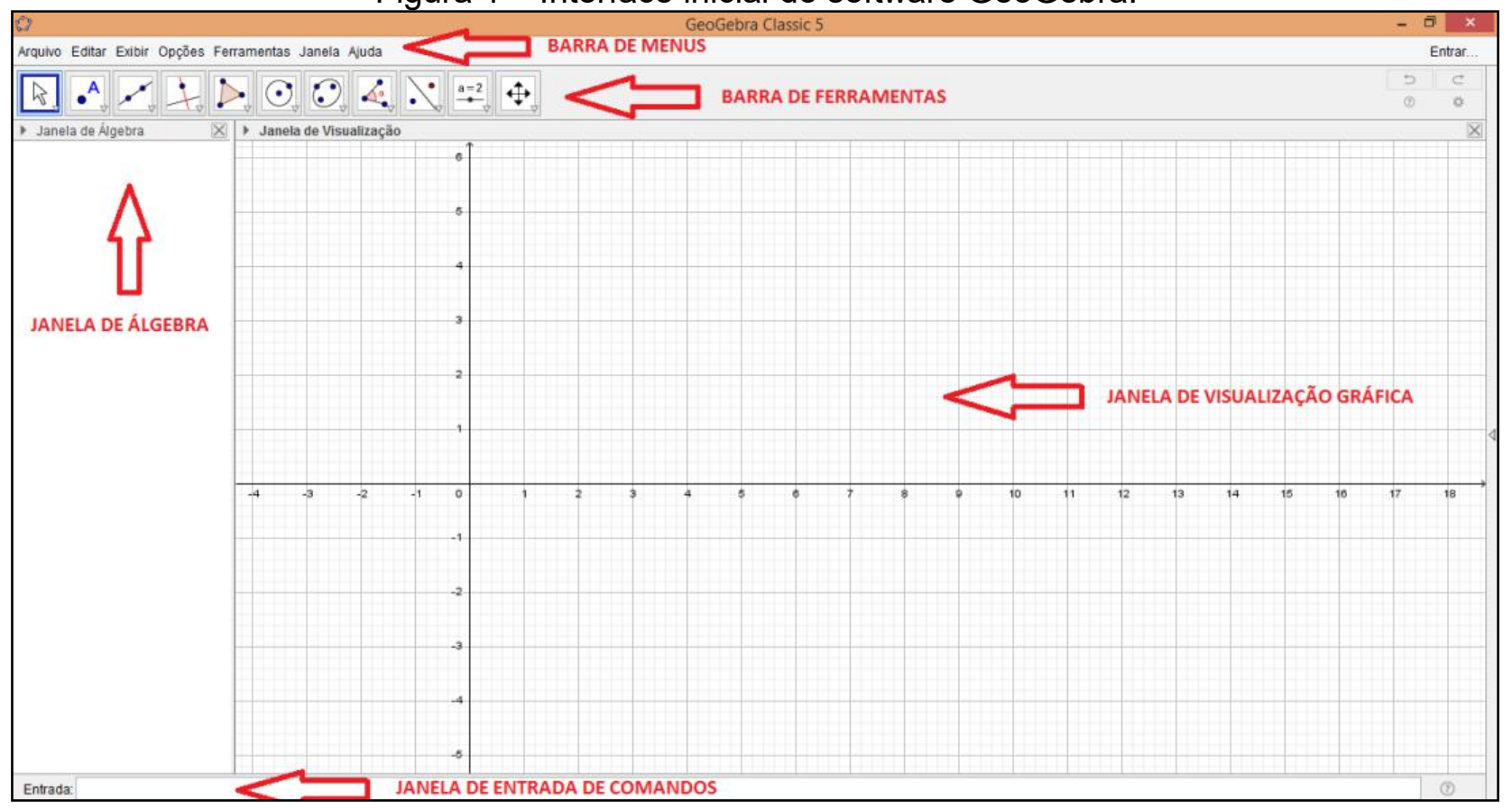

Fonte: Elaboração dos autores (2018). 
Utilizar-se-á como exemplo a função polinomial $f(x)=x^{2}+2 x+1$. Insere-se a função na janela de entrada de comandos. O gráfico da função é apresentado na Figura 2.

Figura $2-$ Gráfico da função $f(x)=x^{2}+2 x+1$.

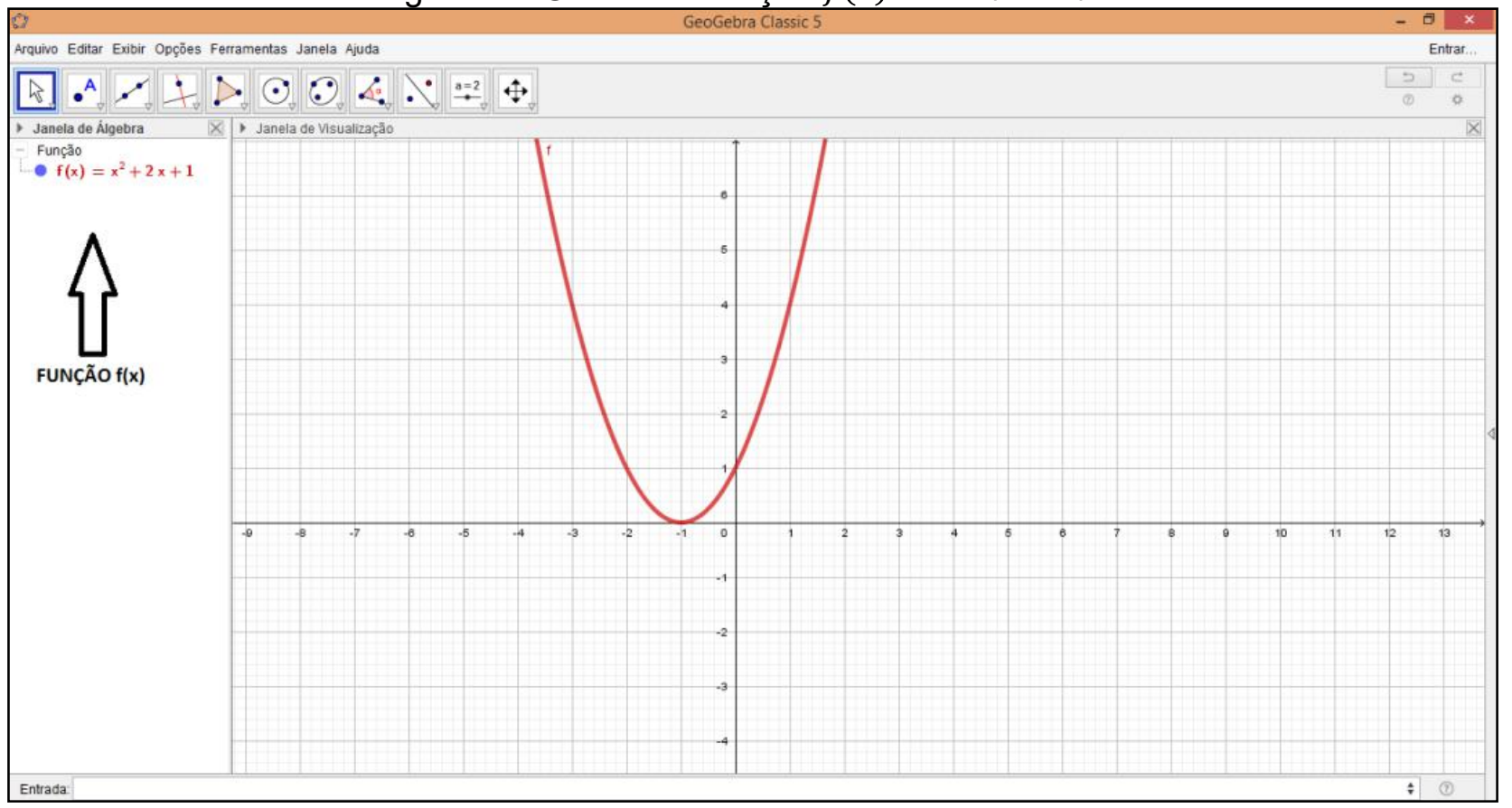

Fonte: Elaboração dos autores (2018).

Na sequência, sugere-se a análise do limite da função $f(x)$ quando $x$ tente a 0 . Para isso, insere-se um ponto $A$ sobre o eixo das abscissas, por meio da barra de ferramentas. Posteriormente, insere-se uma reta perpendicular (reta $g$ ) no ponto $A$ em relação ao eixo das abscissas (isso também é possível fazer com o auxílio da barra de ferramentas). Em seguida, busca-se na barra de ferramentas a opção de "ponto de intersecção entre dois objetos", e, depois se seleciona a reta $g$ e o gráfico da função $f(x)$, criando o ponto $B$ (ponto de intersecção entre a reta $g$ e o gráfico). Na Figura 3 é possível visualizar todos esses passos concluídos.

É possível visualizar na janela de álgebra a função $f(x)$, o ponto $A$ (ponto sobre o eixo das abscissas), o ponto $B$ (intersecção entre a reta $g$ e o gráfico de $f(x)$, além da reta $g$. Sugere-se agora que, utilizando o botão direito do mouse sobre a janela de visualização gráfica, seja usada a opção "zoom" para que a análise seja mais precisa. Em seguida, na barra de ferramentas, usa-se a opção "mover". Posteriormente, com o auxílio do mouse, move-se o ponto $A$ (na janela de visualização gráfica), até o ponto $(-1,0)$, conforme a Figura 4. 
Figura 3 - Reta perpendicular ao eixo das abscissas e ponto de intersecção entre a reta e o gráfico de $f(x)$.

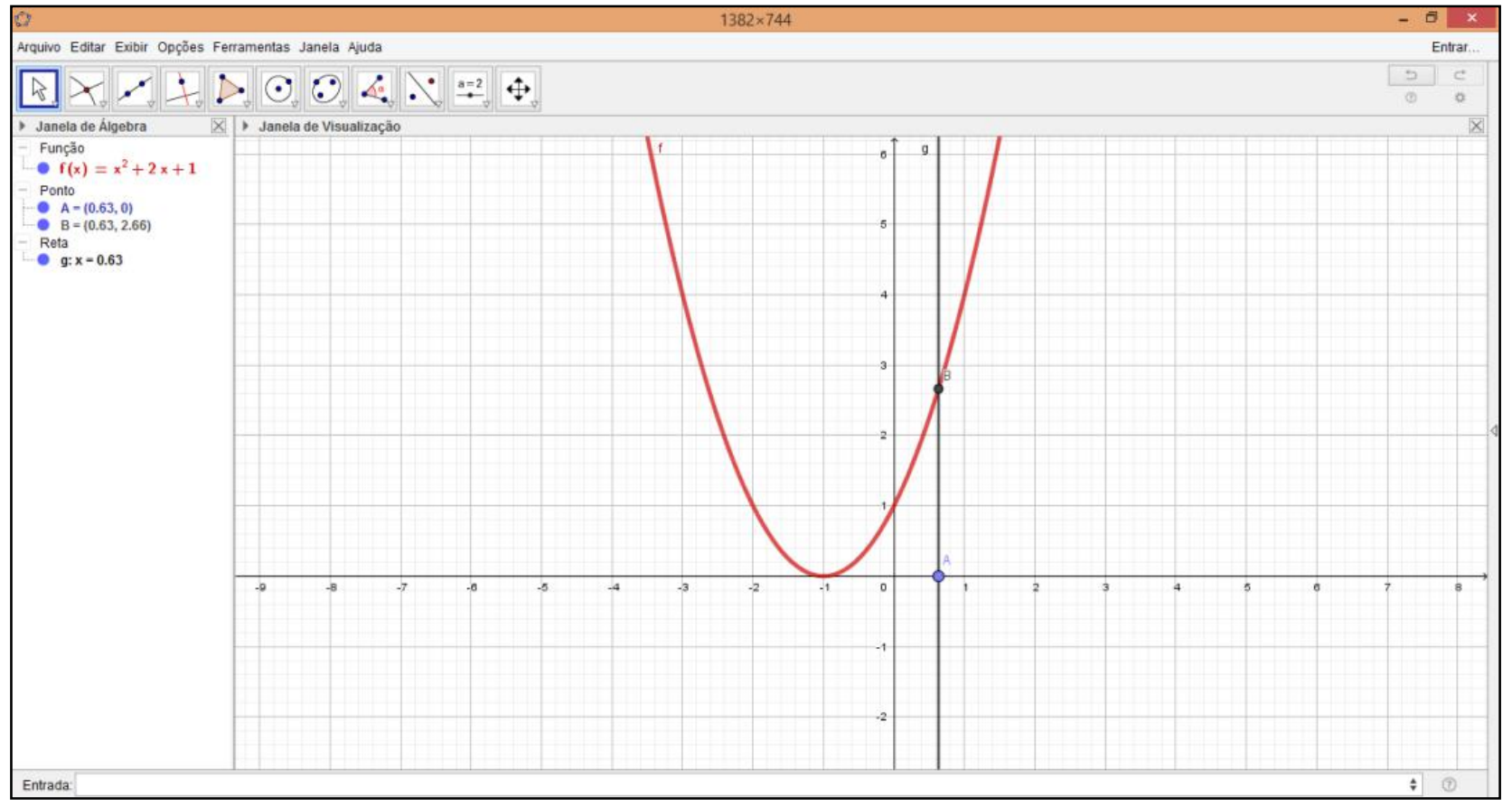

Fonte: Elaboração dos autores (2018).

Figura 4 - Ponto $A(-1,0)$.

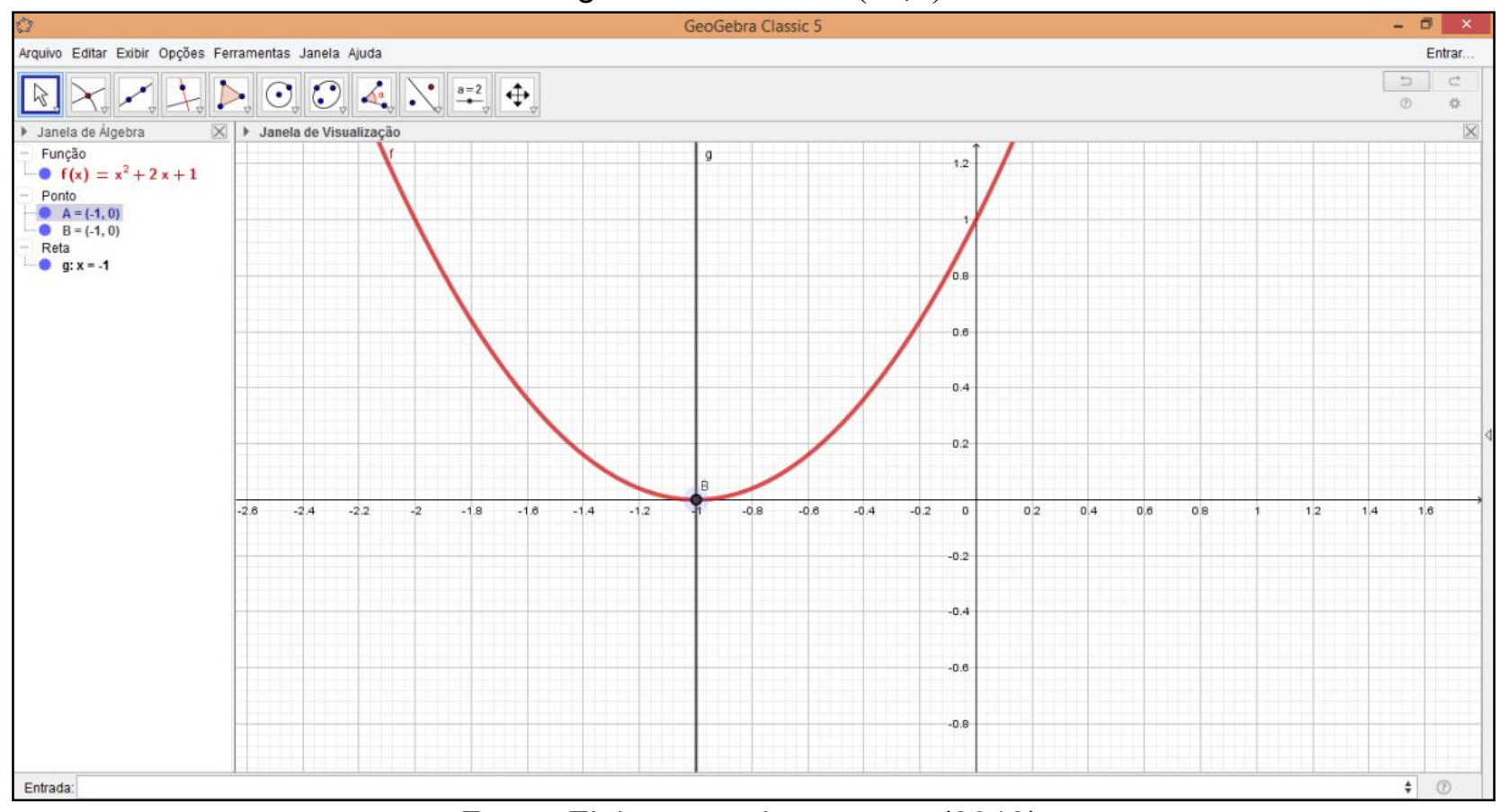

Fonte: Elaboração dos autores (2018).

Na janela de álgebra é possível visualizar que o ponto $B$ também é igual a $(-1,0)$. Como o ponto $B$ está sobre o gráfico da função e representa a intersecção entre o eixo das abscissas e o eixo das ordenadas, é possível concluir que quando $x=-1, y=0$, pois $y$ depende da variável independente $x$. A partir de agora, observa-se somente o valor das coordenadas do ponto $B$. 
Utilizando novamente a ferramenta "mover", sugere-se que o ponto $A$ seja movido lentamente em direção a $x=0$, sem necessariamente chegar a 0 . Repita esse procedimento mais uma vez e observe os valores das coordenadas do ponto $B$ (na janela de álgebra). Aqui, observamos que, quando $x$ tende a 0 pela esquerda, o valor de $y$ se aproxima de 1 . Por exemplo, quando $x=-1 ; y$ chega muito próximo de $1(y=0,81)$, conforme a Figura 5 .

Figura 5-Quando $x$ tende a $0, y$ se aproxima de 1 .

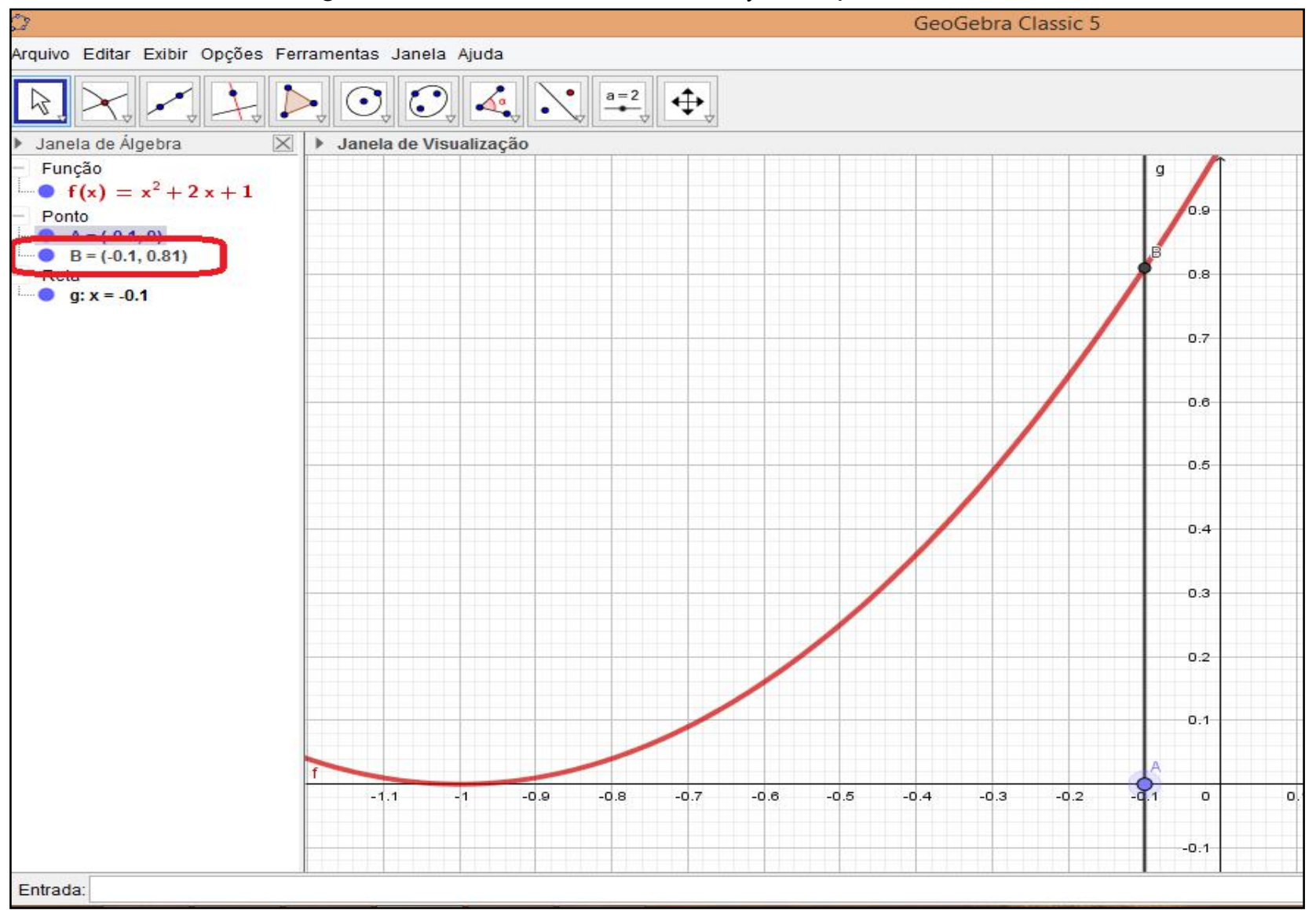

Fonte: Elaboração dos autores (2018).

Portanto, isso sugere que o limite de $f(x)$ quando $x$ tende a 0 pela esquerda é igual a 1 .

Agora, sugere-se que seja feito o mesmo processo quando $x$ tende a 0 pela direita. Para isso, move-se o ponto $A$ para as coordenadas $(1,0)$, conforme a Figura 6.

É possível visualizar no gráfico, bem como na janela de álgebra, através do ponto $B(1,4)$, que para essa função, quando $x=1, y=4$. Novamente, observa-se somente 0 valor das coordenadas do ponto $B$.

Sugere-se agora a movimentação lenta do ponto $A$ em direção a $x=0$, sem necessariamente chegar a 0 . Repetindo o movimento, nota-se (no ponto $B$ ) que quando o valor $x$ tente a 0 , o valor de $y$ se aproxima de 1 . Por exemplo, quando $x=0,1$; o valor de $y$ chega muito próximo de $1(y=1,21)$, conforme a Figura 7 . 
Figura 6 - Ponto $A(1,0)$

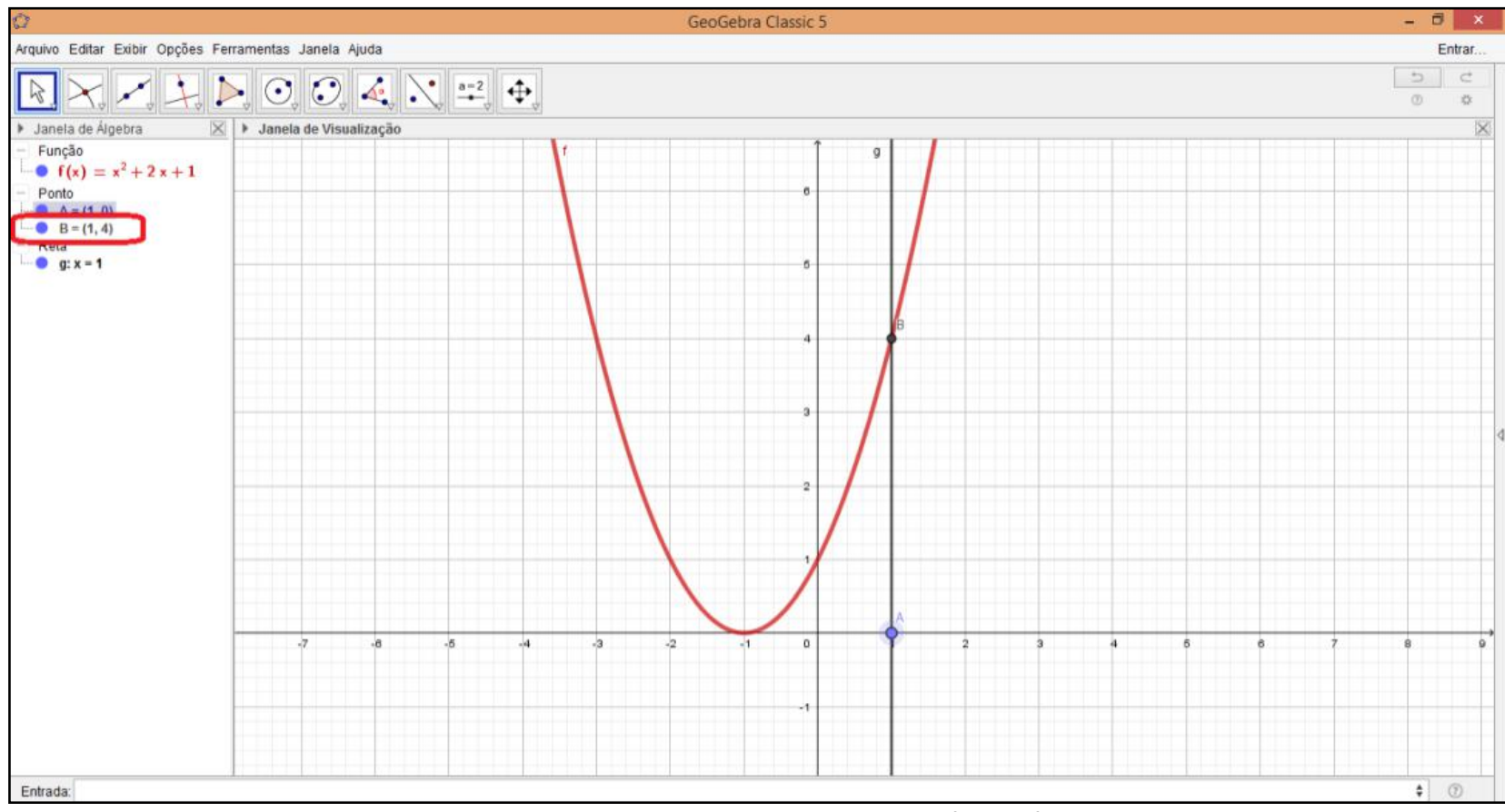

Fonte: Elaboração dos autores (2018).

Figura 7 - Quando $x$ tende a $0, y$ se aproxima de 1 .

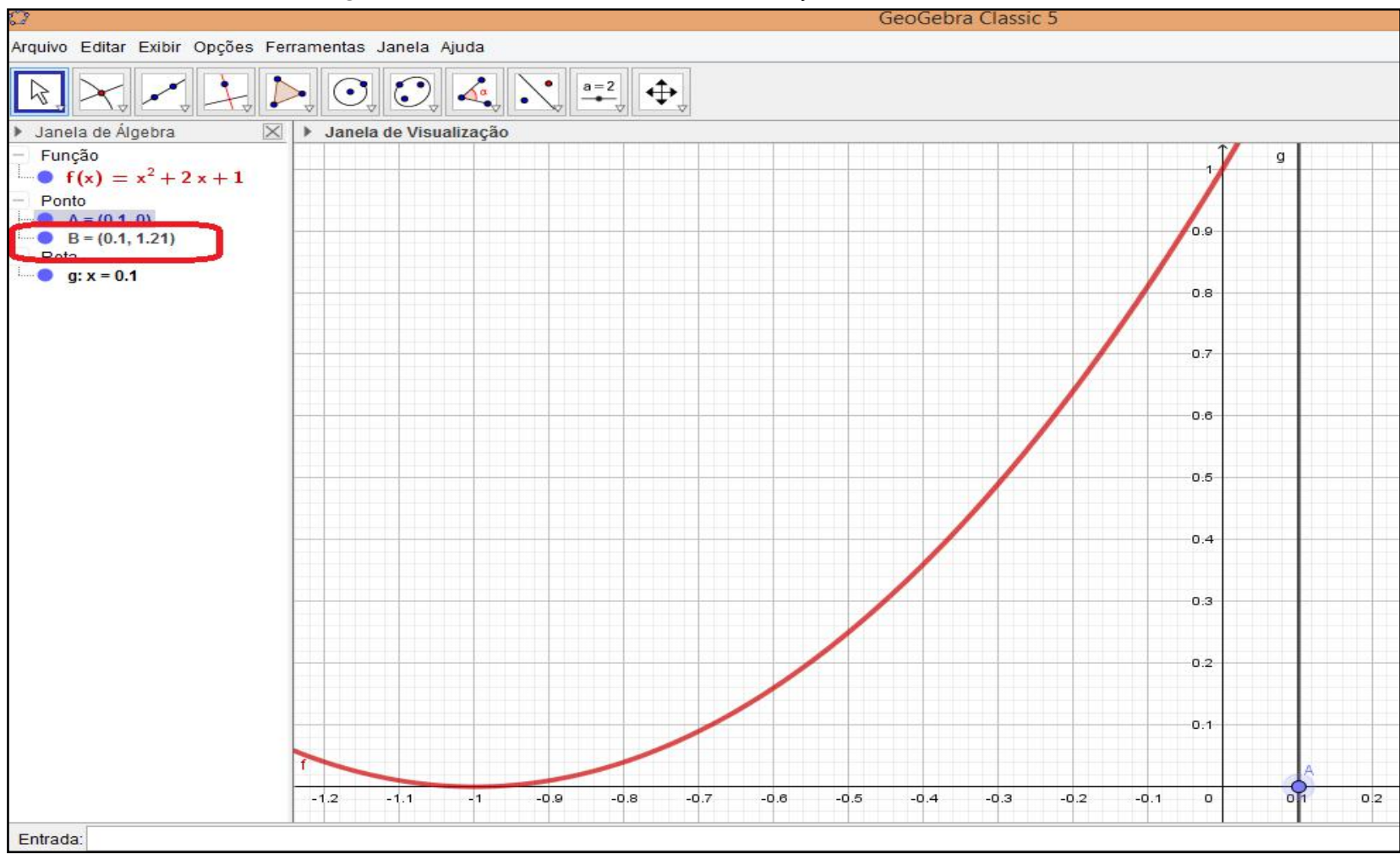

Fonte: Elaboração dos autores (2018).

Portanto, isso sugere que o limite de $f(x)$ quando $x$ tende a 0 pela direita é igual a 1 .

Logo, se o limite de $f(x)$ quando $x$ tende a 0 pela esquerda é igual a 1, e quando tende a 0 pela direita, também é igual 1 , é provável que o limite de $f(x)$ quando $x$ tende a 0 seja 
igual a 1 . Isso pode ser constatado quando se move o ponto $A$ sobre as coordenadas $(0,0)$. Nesse caso, observando as coordenadas do ponto $B$, vemos que quando $x=0, y=1$, conforme a Figura 8.

Figura 8 - Limite de $f(x)$ quando $x$ tende a 0 é igual a 1.

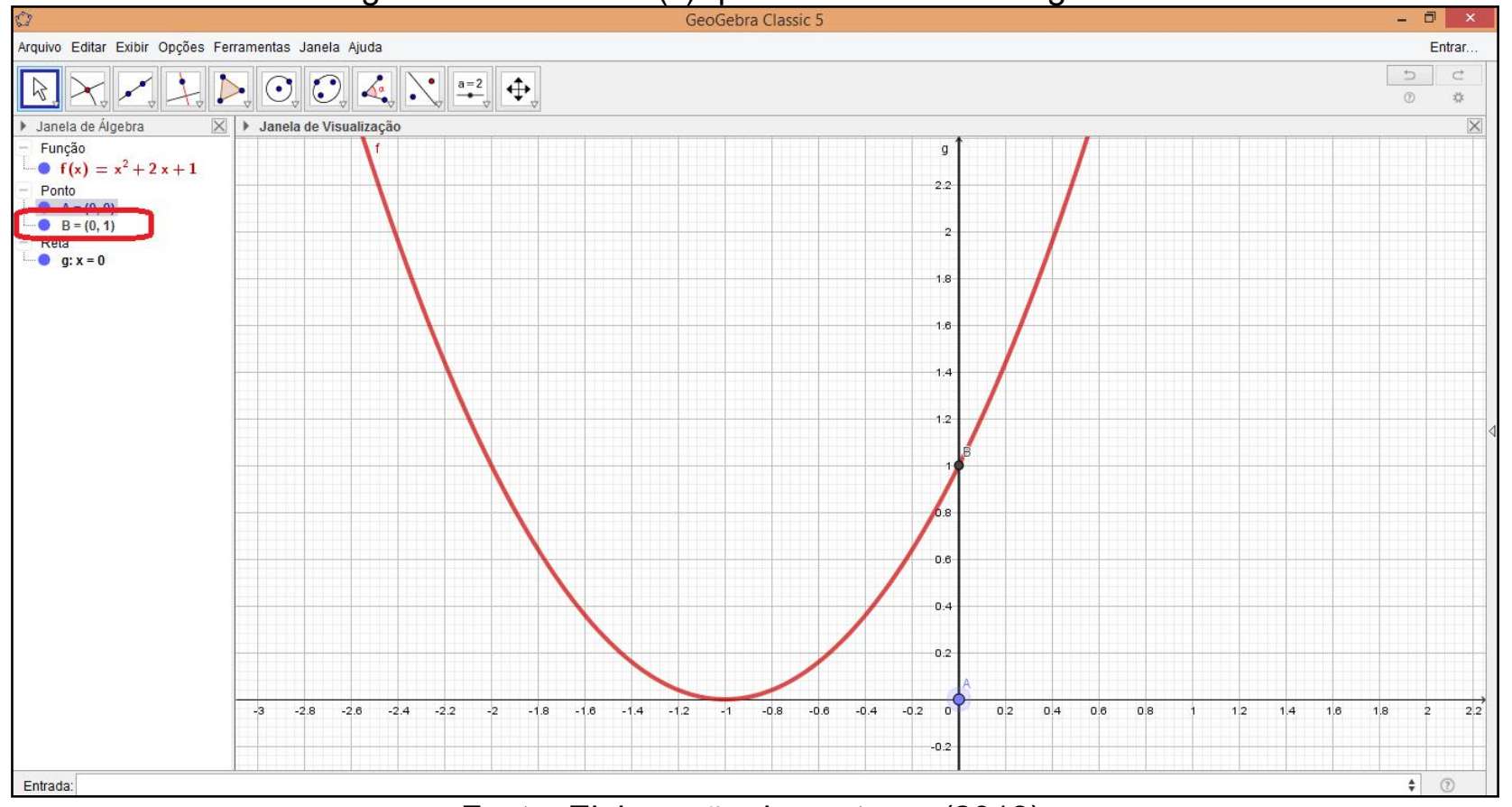

Fonte: Elaboração dos autores (2018).

Além disso, o software GeoGebra possui o recurso do Cálculo Simbólico (CAS). Com essa ferramenta, é possível realizar cálculos auxiliares, como, por exemplo, o limite de uma função. Assim, pode-se realizar a confirmação do resultado obtido através da análise gráfica. Para utilizálo, é necessário buscar na Barra de Menus a opção "exibir" e posteriormente "Cálculo Simbólico (CAS)". Na nova janela que se abrirá, utiliza-se o comando "Limite (<Expressão>, <Valor>)". Substitui-se "<Expressão>" por "f(x)" e "<Valor>" por "0". Em seguida, clica-se na opção "Valor Numérico", na barra de ferramentas. O software confirmará o valor encontrado na análise gráfica, conforme a Figura 9. Portanto, conclui-se que $\lim _{x \rightarrow 0} x^{2}+2 x+1=1$. 
Figura 9 - Utilização da ferramenta "Cálculo Simbólico" para confirmação do resultado da análise gráfica.

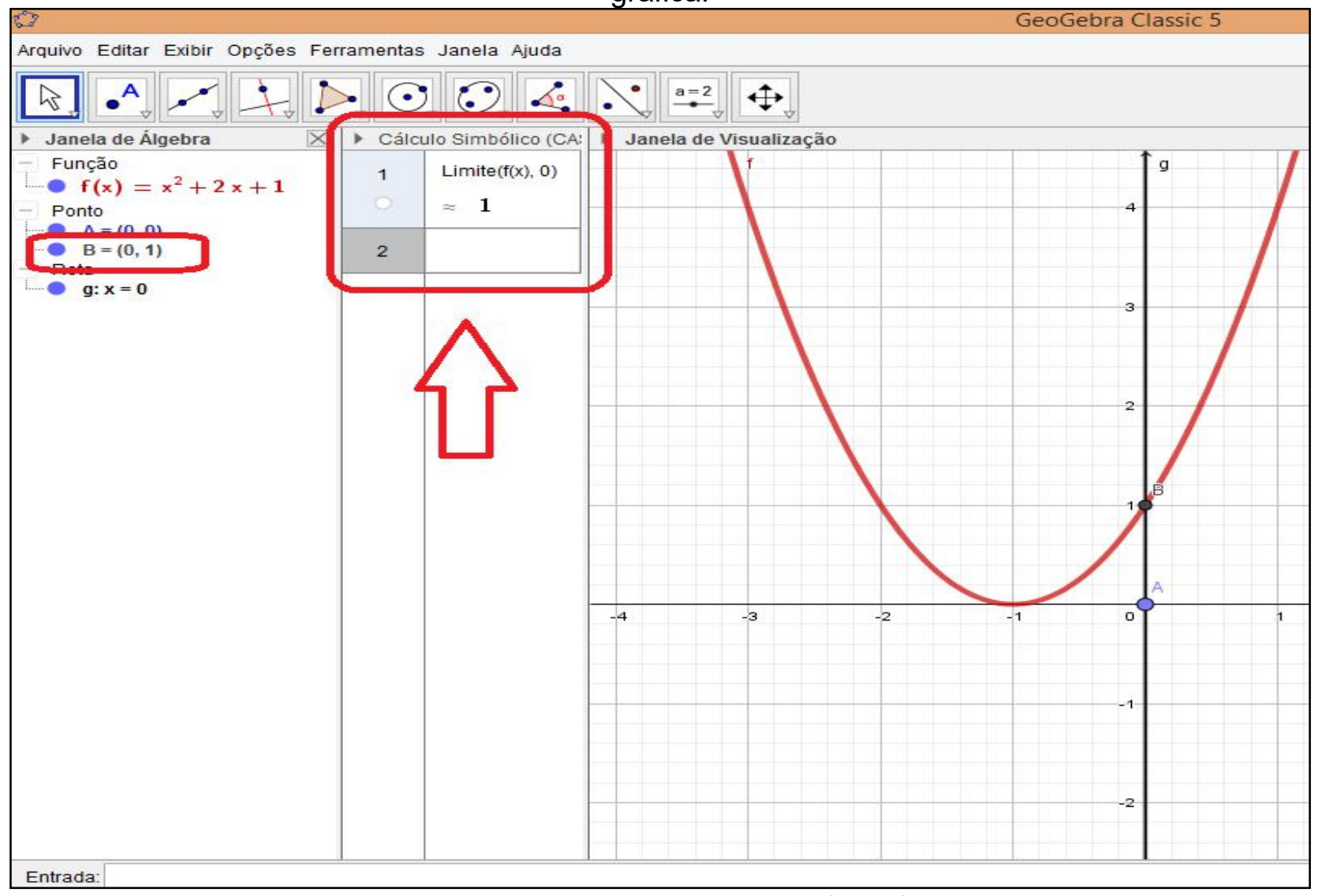

Fonte: Elaboração dos autores (2018).

\section{Análise e discussão da proposta apresentada}

A partir do desenvolvimento da atividade descrita anteriormente, os estudantes podem construir concepções próprias acerca do conceito de limite. Ao analisar graficamente o valor de $x$ tendendo a 0 (sem chegar a 0 ), e o valor de $y$ se aproximando de 1 , tanto pela esquerda quanto pela direita, espera-se que os alunos desenvolvam uma noção intuitiva de limite de uma função.

Definindo intuitivamente o conceito de limite de uma função, os estudantes poderiam pensar como sendo o comportamento de uma função quando sua variável independente tende a determinado valor. Essa tendência está intimamente relacionada ao sentido de aproximação. Outra noção intuitiva poderia estar relacionada ao comportamento da variável dependente em relação à aproximação da variável independente para determinado valor. Após uma discussão coletiva, sugere-se que o docente retome o conceito geral de limite, tal qual descrito por Stewart (2012) ou outro autor.

Nota-se a possibilidade de existirem diferentes respostas para a noção intuitiva de limite, que é o foco da atividade proposta. Isso evidencia o seu teor investigativo, conforme a ideia apresentada no referencial teórico deste trabalho. De acordo com Ponte, Brocardo e Oliveira (2003), a investigação matemática acontece quando o estudante é desafiado a mobilizar recursos 
cognitivos para a formulação de ideias sobre problemas matemáticos. Da mesma forma, é possivel tomar a atividade proposta como aberta, uma vez que é passível de adaptações, estudar outros tipos de funções, bem como recorrer a outros conhecimentos estudados anteriormente, especialmente da Matemática básica.

Outra questão importante a ser abordada é a contribuição das tecnologias digitais no contexto da matemática investigativa. Percebe-se na proposta para o ensino de limite, que os estudantes estão diante da possibilidade de experimentar e simular diferentes situações com o objeto de estudo. Por exemplo, movimentar os objetos na janela de visualização gráfica e verificar as aproximações tanto no gráfico quanto na janela de álgebra, não poderia ser realizado dinamicamente sem um software. Trata-se de ampliar as linguagens e, consequentemente, as formas de interação entre o aluno e conteúdo, assim como mencionam Borba e Penteado (2012) e Richit e Farias (2013).

A abordagem dinâmica do conceito de limite no GeoGebra ajuda a resgatar sua natureza geométrica. Amorim, Sousa e Salazar (2011), ao se referirem ao ensino de Cálculo Diferencial e Integral, destacam que as abordagens pedagógicas têm deixado de lado essa epistemologia, e focado no seu estudo algébrico. Portanto, a proposta busca justamente repensar esse trabalho pedagógico na perspectiva de dinamizar o processo de aprendizagem. Resultados apontados por Richit et al. (2012), Jardim et al. (2015), Silva e Becker (2018) e Fonseca e Henriques (2016) relatam o enriquecimento das discussões ao trabalharem com esta ferramenta. Segundo os autores, foi possível relacionar as diferentes representações sem enfrentar grandes dificuldades, além de observarem maior envolvimento dos alunos.

Todas essas considerações permitem uma relação com a aprendizagem significativa e conceitual (AUSUBEL, 2003). Durante o processo de investigação, o aprendiz está diante de situações em que precisa recorrer a conhecimentos anteriores, desde as noções básicas do plano cartesiano, até o conceito de função e a sua representação gráfica. São conhecimentos prévios, necessários para a aprendizagem do novo conceito. Não há como conceituar limite sem uma gama de conhecimentos anteriores, que irão se entrelaçar, caracterizando a aprendizagem significativa. Da mesma forma, a aprendizagem conceitual acontece a partir do momento em que é feita a generalização da ideia. Ou seja, a partir do estudo desta função, o aluno deve perceber a noção de limite para toda e qualquer função.

\section{Considerações finais}

Diante dos diversos apontamentos realizados no referencial teórico deste trabalho, fica evidente que a realização de atividades de investigação matemática pode ser uma importante aliada na busca pela aprendizagem significativa e conceitual. As TDIC vêm como ferramentas que complementam esse processo a partir da possibilidade de colocar o aprendiz diante de situações que dificilmente estariam disponíveis de outra forma. E, portanto, ao levar em consideração os 
problemas de ensino e aprendizagem em Cálculo Diferencial e Integral, é notável que a incorporação de formas de abordagem inovadoras tem potencial significativo para amenizar esta problemática.

A proposta para o ensino do conceito de limite de uma função apresentada neste trabalho busca fornecer uma possibilidade metodológica para professores da disciplina de Cálculo Diferencial e Integral, tendo como foco justamente estudar a Matemática de forma investigativa para a construção do conceito de forma dinâmica e significativa. Pretende-se, tão breve seja possível, aplicar a metodologia em sala de aula, a fim de se obter resultados práticos.

Por fim, vale destacar que por mais autonomia que o estudante tenha, o trabalho docente continua sendo imprescindível para a aprendizagem. A condição de orientar em um ambiente de aprendizagem dinâmico e coletivo faz do professor a ligação entre o aprendiz e o conhecimento, assim como mencionam Santos e Belline (2016).

\section{Referências}

AMORIM, F. V.; SOUSA, G. C. de; SALAZAR, J. V. Atividades com Geogebra para o ensino de Cálculo. In: CONFERÊNCIA INTERAMERICANA DE EDUCAÇÃO MATEMÁTICA, XIII, 2011, Recife, PE. Anais..., Recife, p. 1-12, 2011. Disponível em: https://xiii.ciaemredumate.org/index.php/xiii ciaem/xiii ciaem/paper/viewFile/1649/749. Acesso em: 15 ago. 2020.

AUSUBEL, D. P. Aquisição e retenção de conhecimentos: uma perspectiva cognitiva. Lisboa: Editora Plátano, 2003.

BARBOSA, J. C. Modelagem Matemática e os professores: a questão da formação. Bolema, Rio Claro, SP, v. 14, n. 15, 2001. Disponível em:

http://www.periodicos.rc.biblioteca.unesp.br/index.php/bolema/article/view/10622. Acesso em: 15 ago. 2020.

BARBOSA, M. A. O insucesso no ensino e aprendizagem na disciplina de Cálculo Diferencial e Integral. 2004. 101f. Dissertação (Mestrado em Educação) - Pontifícia Universidade Católica do Paraná, Curitiba, 2004.

BARUFI, M. C. B. A construção/negociação de significados no curso universitário inicial de Cálculo Diferencial e Integral. São Paulo: FE-USP, 1999.

BORBA, M. C.; PENTEADO, M. G. Informática e Educação Matemática. 5. ed. Belo Horizonte: Autêntica Editora, 2012. Coleção Tendências em Educação Matemática, 2.

BORBA, M. C.; SILVA, R. S. R.; GADANIDIS, G. Fases das tecnologias digitais em Educação Matemática: sala de aula e internet em movimento. 1. ed. Belo Horizonte: Autêntica, 2014.

COSTA, L. S.; OLIVEIRA, C. C. Investigando a produção de significados em Cálculo Diferencial e Integral. Brazilian Electronic Journal of Mathematics, v. 1, n. 1, p. 73-87, 2020. DOI: https://doi.org/10.14393/BEJOM-v1-n1-2020-50262.

FONSECA, V.; HENRIQUES, A. A aprendizagem do conceito de limite de funções com recurso a tarefas exploratórias e ao Geogebra. In: ENCONTRO DE INVESTIGAÇÃO EM EDUCAÇÃO MATEMÁTICA, Évora, Portugal, nov. 2016. Atas..., Universidade de Évora, 2016. 
FUENTE, Á. C. de la; ARMENTEROS, M. G.; MOLL, V. F. Análisis de un proceso de estudio sobre la enseñanza del límite de una función. Bolema, Rio Claro, SP, v. 26, n. 42B, p. 667-690, abr. 2012. DOI: https://doi.org/10.1590/S0103-636X2012000200013.

\section{GARZELLA, F. A. C. A disciplina Cálculo 1: análise das relações entre as práticas} pedagógicas do professor e seus impactos nos alunos. 2013. 275f. Tese (Doutorado em Educação) - Faculdade de Educação, Universidade Estadual de Campinas, Campinas, 2013.

GONÇALVES, D. C.; REIS, F. da S. Atividades investigativas de aplicações das derivadas utilizando o GeoGebra. Bolema, Rio Claro, SP, v. 27, n. 46, p. 417-432, ago. 2013. DOI: http://dx.doi.org/10.1590/S0103-636X2013000300006.

JARDIM, D. F.; SILVA, J. M. PEREIRA, M. M.; SOARES JUNIOR, E. A. NEPOMUCENA, T. V. Estudando limites com o GeoGebra. Vozes dos Vales, Diamantina, MG, ano IV, n. 8, p. 1-19, out. 2015. Disponível em: http://site.ufvim.edu.br/revistamultidisciplinar/files/2015/11/Deborah.pdf. Acesso em: 17 abr. 2020.

LUCKESI, C. C. Gestão democrática da escola, ética e sala de aula. Revista ABC Educatio, São Paulo, SP, v. 64, n. 64, p. 30-33, mar. 2007.

MOTTA, M. S.; ROLIM, M. R. L. B.; SILVEIRA, I. F.; ARAÚJO JUNIOR, C. F. O uso de tecnologias educacionais no desenvolvimento da aprendizagem matemática. Revista Ceciliana, Santos, SP, ano 22, n. 32, p. 153-162, 2011.

ONUCHIC, L. de la R. Ensino-Aprendizagem de Matemática Através da Resolução de Problemas. In: BICUDO, Maria Aparecida Viggiani (Org.). Pesquisa em Educação Matemática: concepções e perspectivas. São Paulo: Editora UNESP, p. 199-218, 1999.

PAGANI, E. M. L.; ALEVATTO, N. S. G. Ensino e aprendizagem de Cálculo Diferencial e Integral: um mapeamento das teses e dissertações produzidas no Brasil. Revista Vidya, Santa Maria, RS, v. 34, n. 2, p. 61-74, 2014.

PONTE, J. P. da; BROCARDO, J.; OLIVEIRA, H. Investigações Matemáticas na Sala de Aula. Belo Horizonte: Autêntica, 2003.

RESENDE, W. M. O ensino de Cálculo: Dificuldades de Natureza Epistemológica. 2003. 468f. Tese (Doutorado em Educação) - Faculdade de Educação, Universidade de São Paulo, 2003.

RICHIT, A.; BENITES, V. C.; ESCHER, M. A.; MISKULIN, R. G. S. Contribuições do software GeoGebra no estudo de cálculo diferencial e integral: uma experiência com alunos do curso de geologia. Revista do Instituto GeoGebra Internacional de São Paulo. v. 1, n. 1, p. 90-99, 2012.

RICHIT, A.; FARIAS, M. M. Cálculo diferencial e integral e tecnologias digitais: perspectivas de exploração no sofware GeoGebra. In: CONGRESO DE EDUCACIÓN MATEMÁTICA DE AMÉRICA CENTRAL Y EL CARIBE, 1., 2013, Santo Domingo, República Dominicana. Anais... Red de Educación Matemática de América Central y El Caribe, 2013.

SANTOS, C. H. M. dos; BELLINI, W. Investigações matemáticas em sala de aula: Contribuições de uma Tarefa Investigativa no $1^{\circ}$ Ano do Ensino Médio. In: ENCONTRO NACIONAL DE EDUCAÇÃO MATEMÁTICA, XII, jul. 2016, São Paulo, SP. Anais..., SBEM, 2016.

SILVA, A. J. da; BECKER, F. Processos da construção de conceitos matemáticos com o Geogebra: o caso do limite de funções reais. Projeção e Docência, Brasília, v. 9, n. 2, p. 188-198, 2018. Disponível em: http://revista.faculdadeprojecao.edu.br/index.php/Projecao3/article/view/1131. Acesso em: 15 ago. 2020. 
SILVA, K. A. P. da; VERTUAN, R. E. Um estudo sobre as intervenções docentes em contextos de atividades investigativas no âmbito de aulas de Matemática do Ensino Superior. Ciência \& Educação, Bauru, v. 24, n. 2, 501-516. abr./jun. 2018. DOI: https://doi.org/10.1590/1516$\underline{731320180020015}$.

SKOVSMOSE, O. Educação Matemática Crítica: a questão da democracia. Campinas, SP: Papirus, 2001. Coleção Perspectivas em Educação Matemática.

STEWART, J. Cálculo. V. 1, São Paulo: Cengage Learning, 2012.

VIEIRA, A. R. L.; RIOS, P. P. S.; VASCONCELOS, C. A. de. Aprendizagem significativa e a estratégia do uso de mapas conceituais no ensino de Cálculo Diferencial e Integral no curso de Bacharelado em Engenharia Elétrica. Revista de Ensino de Engenharia, v. 39, n. 2, p. 93-102, 2019.

WISLAND, B.; FREITAS, M. C. D.; ISHIDA, C. Y. Desempenho acadêmico dos alunos em curso de Engenharia e Licenciatura na disciplina de Cálculo I. Iberoamerican Journal of Industrial Engineering, Florianópolis, SC, v. 6, n. 11, p. 94-112, 2014. 